\title{
Higher locoregional recurrence in hormone receptor-negative breast cancer patients with residual disease after neoadjuvant treatment undergoing breast conservation surgery
}

Hsu-Huan Chou ( $\nabla$ b9002009@cgmh.org.tw )

Chang Gung Memorial Hospital https://orcid.org/0000-0002-0948-1141

Wei-Shan Chung

Chang Gung Memorial Hospital

Rong-Yao Ding

Chang Gung Memorial Hospital

Wen-Ling Kuo

Chang Gung Memorial Hospital

Chi-Chang Yu

Chang Gung Memorial Hospital

Hsiu-Pei Tsai

Chang Gung Memorial Hospital

Shih-Che Shen

Chang Gung Memorial Hospital

Chia-Hui Chu

Chang Gung Memorial Hospital

Yung-Feng Lo

Chang Gung Memorial Hospital

Shin-Cheh Chen

Chang Gung Memorial Hospital

Research article

Keywords: Breast cancer, neoadjuvant chemotherapy, locoregional recurrence, hormone receptor

Posted Date: July 23rd, 2020

DOI: https://doi.org/10.21203/rs.3.rs-41619/v1

License: (c) (1) This work is licensed under a Creative Commons Attribution 4.0 International License.

Read Full License 
Version of Record: A version of this preprint was published on March 23rd, 2021. See the published version at https://doi.org/10.1186/s12893-021-01158-7. 


\section{Abstract \\ Background}

Neoadjuvant chemotherapy (NAC) has been the standard treatment for locally advanced breast cancer for the purpose of downstaging or for conversion from mastectomy to breast conservation surgery (BCS). Locoregional recurrence (LRR) rate is still high after NAC. The aim of this study was to determine predictive factors for locoregional recurrence (LRR) in breast cancer patients in association with the operation types after NAC.

\section{Methods}

Between 2005 and 2017, 1047 breast cancer patients underwent BCS or mastectomy after NAC in Chang Gung Memorial Hospital, Linkou. We obtained data regarding patient and tumor characteristics, chemotherapy regimens, clinical tumor response, tumor subtypes and pathological complete response $(\mathrm{pCR})$, type of surgery, and recurrence.

\section{Results}

The median follow-up time was 45.1 months (range 0.1-160.3 months). The mean initial tumor size was $4.89 \mathrm{~cm}(\mathrm{SD} \pm 2.95 \mathrm{~cm})$. Of the 1047 NAC patients, $232(22.2 \%)$ achieved pCR. The BCS and mastectomy rates were $41 \%$ and $59 \%$, respectively. Overall, 240 patients experienced tumor recurrence $(22.9 \%)$. Thirtyfive cases of LRR (14.3\%) were noted following BCS, of which $4.3 \%$ achieved pCR. Multivariate analysis indicated that independent factors for the prediction of LRR included hormone receptor negative/human epidermal growth factor receptor 2 positive (HR-/HER2+) subtype, HR-/HER2- subtype, and failure to achieve pCR. Further investigation according to the molecular subtype showed that following BCS, HR-/HER2 + non-pCR group had significantly increased LRR compared with the HR+/HER2 + pCR group (22.2\% vs $6.3 \%, p<0.05)$, and the HR-/HER2-non-pCR group had significantly increased LRR compared with the HR-/HER2-pCR group (0\% vs $20.4 \%, p<0.005)$.

\section{Conclusion}

Pathological response after NAC is related to the risk of developing LRR. The LRR rate was higher in nonpCR patients after NAC, especially in hormone receptor-negative patients undergoing BCS. Therefore, both the pathological response status and molecular subtype should be carefully considered when considering candidates for BCS after NAC.

\section{Background}


Neoadjuvant chemotherapy (NAC) has generally been accepted as the standard treatment for locally advanced breast cancer. With the aim of making non-operable breast cancer operable, NAC increases the number of breast conservative therapy (BCS) candidates by downsizing locally advanced tumor $[1,2]$. Hence, patients undergoing BCS after NAC might have a more favorable cosmetic outcome and quality of life[3].

Despite the fact that increased use of NAC has facilitated the treatment of breast cancer, long-term optimized outcomes and treatment effects are concerning. In their recently published meta-analysis on NAC and adjuvant chemotherapy, Asselain and colleagues reported that NAC has a higher local recurrence rate than adjuvant chemotherapy in patients with locally advanced tumors[4]. Similar results were reported in a meta-analysis by Mauri in 2005[5]. The frequency of BCS, defined as lumpectomy with whole breast irradiation, is higher after the use of NAC compared with adjuvant chemotherapy after surgery.

The long-term survival rate among early breast cancer women without NAC who undergo BCS was the same as that among women who undergo radical mastectomy [6-7]. However, several studies have demonstrated no significant difference in long-term survival or local recurrence rates between women who underwent BCS and those who underwent radical mastectomy after NAC[8-9]. Although predictive factors for local recurrence in these NAC-treated patient groups have not yet been clarified yet, the higher local recurrence risk of NAC than of adjuvant chemotherapy is controversial.

NAC is increasingly used in breast cancer therapy, and clinic-pathological subtypes, such as estrogen receptor positive (ER), progesterone receptor positive (PR), and human epidermal growth factor receptor 2 positive (HER-2), can provide prognostic information regarding the risk of local regional recurrence after NAC. Furthermore, pathological complete remission ( $p C R$ ) after NAC can significantly affect the prognosis of breast cancer $[10,11]$. We would like to investigate these attributed factors for recurrence reported in retrospective studies on NAC. Furthermore, once the PCR-achieved status is confirmed, the omission of mastectomy is feasible. Patients receiving NAC, in total, have higher BCS rate in literature. We investigated these clinic-pathological attributed factors in association with the operation types after NAC.

\section{Methods}

The study was a retrospective cohort study and was approved by ethics committee of our institution and institutional review board number was 1711150042 . Female patients diagnosed with histologically proven unilateral invasive breast cancer who received neo-adjuvant chemotherapy (NAC) and underwent mastectomy or breast conserving surgery at Chang Gung Memorial Hospital, Linkou, between 2005 and 2017 were enrolled. Exclusion criteria were inflammatory cancer, the initial presence of distant metastasis and loss of clinical information. Clinical staging was determined through physical examination, mammography, ultrasonography of the breast and axillary lymph nodes, a bone scan, and a whole body computed tomography (CT) scan. Histological Diagnosis was confirmed by the specimen of core needle 
biopsy and abnormal axillary lymph node was routinely evaluated by fine needle aspiration. Clinical information including age, tumor histology, molecular subtype, TNM stage, Scarff-Bloom-Richardson (SBR) grade, NAC regimens, post-NAC response status, operation type, and locoregional recurrence were collected. Chemotherapy regimens included anthracycline-based and taxane-based regimens and the target therapy for HER2 contained trastuzumab and pertuzumab for partial patients. Dose modifications were based on blood cell counts and adverse events. Operation including mastectomy or BCS and axillary sentinel lymph node biopsies or axillary lymph node dissections according to clinical posttreatment evaluation were performed 2-4 weeks after NAC was completed. The indication for postoperative radiotherapy included all patients after BCS and the patients receiving mastectomy with initial T3 stage (tumor size $>5 \mathrm{~cm}$ ) and initial N2 stage or residual positive lymph node after NAC. Almost patients received postoperative adjuvant therapy, including hormone therapy, chemotherapy, and target therapy, according to their clinicopathological factors. Analysis of estrogen receptor, progesterone receptor, HER2 expression and the definition of pCR were described below. The analysis was performed using immunohistochemistry $(\mathrm{IHC})$ staining techniques on pretreatment core needle biopsy specimens. For ER and PR, the positivity in $>1 \%$ of tumor cells was defined as expression. Hormone receptor (HR) positive was defined as ER or PR positive while HR negative was categorized as both negative ER and PR. The definition of positive HER2 was IHC staining with a score of $3+$ or a positive fluorescence in situ hybridization (FISH) test with IHC staining with a score of 2+. pCR was defined as no residual invasive cancer cells in the breast and axillary lymph nodes (ypTO/ypTisN0).

\section{Statistical analysis}

Numerical data were compared using a Student's t-test and presented as the mean + standard deviation (SD) and a two-tailed $p$-value $<0.05$ was considered to be statistically significant. Pearson's chi-square test ( $\chi^{2}$ test) was used to compare the differences in the proportions of categorical data. A log-rank test and the Kaplan-Meier method were used for survival analysis. For multivariate analysis of prognostic factors, logistic regression was used. All analyses were performed using SPSS 22.0 for Windows (SPSS, Chicago, IL).

\section{Results}

During the study period, we enrolled 1047 consecutive patients with breast cancer who were treated with NAC followed by surgery and adjuvant therapy. The median follow-up time was 45.1 months (range 0.1160.3 months). The mean initial tumor size was $4.89 \mathrm{~cm}(\mathrm{SD} \pm 2.95 \mathrm{~cm})$. The T2 stage accounted for 582 patients (55.6\%), T3 for 205 patients (19.6\%), and T4 for 220 patients $(21.0 \%)$. For the clinical lymph node status, 79 patients (7.5\%) were N0, $543(51.9 \%)$ were N1, $425(40.6 \%)$ were N2. Overall, $1028(98 \%)$ were diagnosed with invasive ductal carcinoma, of whom 79 patients $(7.4 \%)$ had tumor SBR grade 1, 405 $(38.7 \%)$ had grade 2 for, and 466 (44.5\%) had grade 3 . According to IHC staining, $413(39.4 \%)$ patients were ER-negative, while 634 patients (60.6\%) were ER-positive; 520 (49.7\%) patients were PR-negative, while 527 (50.3\%) were PR-positive; and 466 (44.5\%) patients were HER-2 positive. The triple negative subtype (hormone receptor negative (HR-)/HER2-) accounted for $15.5 \%(n=162)$ of patients, and the 
hormone receptor positive (HR+)/HER2- subtype accounted for $40.0 \%(n=419)$. Regimens for NAC was prescribed according to the physician's preference. A total of 603 patients $(57.6 \%)$ were prescribed anthracycline-based agents combined with taxane-based regimens, while 325 patients $(31.0 \%)$ received chemotherapy agents combined with target therapies, of whom 68 patients received dual blockade therapy for HER2 + disease. All patients underwent surgery after NAC; the BCS rate was $41.3 \%(n=432)$, and the mastectomy rate was $58.7 \%(n=615)$. The overall pCR rate was $22.2 \%(n=232)$, while the nonpCR rate was $77.8 \%(n=815)$ (Table 1$)$. 
Table 1

Clinicopathological characteristics of the study population

\begin{tabular}{|c|c|}
\hline Parameter & No. of cases \\
\hline Age (years), median (IQR) & $49(14)$ \\
\hline Tumor size (cm), median (IQR) & $4.1(2.3)$ \\
\hline Clinical T stage & $40(3.8 \%)$ \\
\hline T1 & $582(55.6 \%)$ \\
\hline $\mathrm{T} 2$ & $205(19.6 \%)$ \\
\hline T3 & $220(21.0 \%)$ \\
\hline \multicolumn{2}{|l|}{$\mathrm{T} 4$} \\
\hline Clinical lymph node status & $79(7.5 \%)$ \\
\hline NO & $543(51.9 \%)$ \\
\hline N1 & $425(40.6 \%)$ \\
\hline \multicolumn{2}{|l|}{ N2 } \\
\hline Histologic type & $1028(98.2 \%)$ \\
\hline Invasive ductal carcinoma & $4(0.4 \%)$ \\
\hline Mucinous carcinoma & $10(1.0 \%)$ \\
\hline Invasive lobular carcinoma & $5(0.5 \%)$ \\
\hline \multicolumn{2}{|l|}{ Invasive micropapillary carcinoma } \\
\hline SBR grade & $77(7.4 \%)$ \\
\hline 1 & $405(38.7 \%)$ \\
\hline 2 & $466(44.5 \%)$ \\
\hline 3 & $99(9.5 \%)$ \\
\hline \multicolumn{2}{|l|}{ Unknown } \\
\hline ER status & $413(39.4 \%)$ \\
\hline Negative & $634(60.6 \%)$ \\
\hline Positive & \\
\hline
\end{tabular}

ER, estrogen receptor; HR, hormone receptor; HER-2, human epidermal growth factor receptor 2; $\mathrm{pCR}$, pathological complete response; PR, progesterone receptor 


\begin{tabular}{|c|c|}
\hline Parameter & No. of cases \\
\hline PR status & $520(49.7 \%)$ \\
\hline Negative & $527(50.3 \%)$ \\
\hline \multicolumn{2}{|l|}{ Positive } \\
\hline HER2 status & $581(55.5 \%)$ \\
\hline Negative & $466(44.5 \%)$ \\
\hline \multicolumn{2}{|l|}{ Positive } \\
\hline Subtype & $419(40.0 \%)$ \\
\hline HR+/HER2- & 237 (22.6\%) \\
\hline $\mathrm{HR}+/ \mathrm{HER} 2+$ & $229(21.9 \%)$ \\
\hline HR-/HER2+ & $162(15.5 \%)$ \\
\hline \multicolumn{2}{|l|}{ HR-/HER2- } \\
\hline Surgery & $615(58.7 \%)$ \\
\hline Mastectomy & $432(41.3 \%)$ \\
\hline \multicolumn{2}{|l|}{ Breast conserving surgery } \\
\hline Neoadjuvant Regimens & $37(3.5 \%)$ \\
\hline Anthracycline only & $82(7.8 \%)$ \\
\hline Taxane only & $603(57.6 \%)$ \\
\hline Antracycline + Taxane & $325(31.0 \%)$ \\
\hline \multicolumn{2}{|c|}{ Chemotherapy + target therapy } \\
\hline Pathological response & $232(22.2 \%)$ \\
\hline $\mathrm{pCR}$ & $815(77.8 \%)$ \\
\hline \multicolumn{2}{|l|}{ non-pCR } \\
\hline
\end{tabular}

Clinical T stage $(p<0.0001)$, clinical N stage $(p<0.0001)$, and molecular subtype $(p<0.0001)$ were predictors of $\mathrm{pCR}$ in univariate analysis (Table 2). Multivariate analysis found similar results, including clinical T1 stage $(p<0.001)$ and clinical T2 $(p=0.024)$ as predictors of $p C R$ compared with clinical T4 stage. Clinical N0 $(p=0.009)$ and clinical N1 $(p<0.0001)$ were also predictors of pCR compared with clinical N2 stage. With regard to subtype classification, HR-/HER2+ $(p<0.0001)$ and HR-/HER2- $(p<$ 0.0001 ) were predictors of pCR (Table 3 ). 
Table 2

Comparison between patients pCR and non-pCR.

\begin{tabular}{|c|c|c|c|}
\hline Parameter & $\begin{array}{l}\text { PCR } \\
(n=232)\end{array}$ & $\begin{array}{l}\text { non-pCR } \\
(n=815)\end{array}$ & p-value \\
\hline Age (years) & $132(23.0 \%)$ & $443(77.0 \%)$ & 0.493 \\
\hline$<50$ & $100(21.2 \% \%)$ & $372(78.8 \%)$ & \\
\hline \multicolumn{4}{|l|}{$>50$} \\
\hline Clinical T stage & $17(42.5 \%)$ & $23(57.5 \%)$ & $<0.0001$ \\
\hline T1 & $149(25.6 \%)$ & $433(74.4 \%)$ & \\
\hline T2 & $39(19.0 \%)$ & $166(81.0 \%)$ & \\
\hline T3 & $27(12.3 \%)$ & $193(87.7 \%)$ & \\
\hline \multicolumn{4}{|l|}{$\mathrm{T} 4$} \\
\hline Clinical lymph node status & $19(24.1 \%)$ & $60(75.9 \%)$ & $<0.0001$ \\
\hline No & $164(30.2 \%)$ & $379(69.8 \%)$ & \\
\hline N1 & 49 (11.5\%) & $376(88.5 \%)$ & \\
\hline \multicolumn{4}{|l|}{ N2 } \\
\hline Subtype & $27(6.4 \%)$ & $392(93.6 \%)$ & $<0.0001$ \\
\hline HR+/HER2- & $66(27.8 \%)$ & $171(21.0 \%)$ & \\
\hline HR+/HER2+ & $88(37.9 \%)$ & $141(61.6 \%)$ & \\
\hline HR-/HER2+ & $51(31.5 \%)$ & $111(68.5 \%)$ & \\
\hline \multicolumn{4}{|l|}{ HR-/HER2- } \\
\hline
\end{tabular}


Table 3

Multivariate analysis of factors predicting pCR after NAC

\begin{tabular}{|llll|}
\hline Parameter & Odds ratio & $95 \%$ confidence interval & p-value \\
\hline Clinical T stage & 4.400 & $1.918-10.095$ & $<0.001$ \\
T1 vs T4 & 1.763 & $1.079-2.879$ & 0.024 \\
T2 vs T4 & 1.280 & $0.720-2.278$ & 0.401 \\
T3 vs T4 & & & \\
Clinical N stage & 2.390 & $1.245-4.589$ & 0.009 \\
N0 vs N2 & 2.847 & $1.941-4.173$ & $<0.0001$ \\
N1 vs N2 & & & $<0.0001$ \\
\hline $\begin{array}{l}\text { Subtype } \\
\text { HR-/HER2 + vs HR+/HER2- }\end{array}$ & 7.469 & $4.783-11.665$ & $<0.0001$ \\
HR-/HER2- vs HR+/HER2- & 6.761 & & \\
\hline $\begin{array}{l}\text { HR, hormone receptor; HER-2, human epidermal growth factor receptor 2; NAC, neoadjuvant } \\
\text { chemotherapy; pCR, pathological complete response }\end{array}$ & \\
\hline
\end{tabular}

During the follow up period, the recurrence rate was $22.9 \%(n=240)$, of which $8.6 \%(n=90)$ was locoregional recurrence (LRR) and $16.0 \%$ was distal metastasis. By the conclusion of this study, 143 $(13.7 \%)$ patients had died from breast cancer and 19 (1.8\%) patients had died from other causes. Furthermore, on univariate analysis of factors affecting LRR, we noted that clinical T4 stage $(T 4, p=$ $0.029)$, clinical $N 2$ stage $(p=0.001)$, SBR grade $3(p=0.007)$, negative ER $(p=0.001)$, negative $P R(p=$ $0.002)$, HR- subtype $(p=0.037)$, and non-pCR $(p<0.005)$ were predictors of LRR (Table 4$)$. Comparing between patients who underwent BCS and those who underwent mastectomy revealed no significant difference in the overall recurrent rate of the two groups $(p=0.633)$. In multivariate analysis, only negative hormone receptor subtypes and non-pCR were found to be predictors of LRR (Table 5). Locoregional free survival rate (LRRFS) in the BCS and mastectomy groups are shown in Fig. $1(p=0.893)$. The effect of pCR on LRRFS is demonstrated in Fig. $2(p=0.006)$. 
Table 4

Factors predicitng LRR after NAC

\begin{tabular}{|c|c|c|c|}
\hline Parameter & LRR & No LRR & p-value \\
\hline Age (years) & $49(8.5 \%)$ & $526(91.5 \%)$ & 0.925 \\
\hline$<50$ & $41(8.7 \%)$ & $431(91.3 \%)$ & \\
\hline \multicolumn{4}{|l|}{$>50$} \\
\hline Clinical T stage & $5(12.5 \%)$ & $35(87.5 \%)$ & 0.029 \\
\hline T1 & $40(6.9 \%)$ & $542(93.1 \%)$ & \\
\hline $\mathrm{T} 2$ & $16(7.8 \%)$ & $189(92.2 \%)$ & \\
\hline T3 & $29(13.2 \%)$ & $191(86.8 \%)$ & \\
\hline \multicolumn{4}{|l|}{ T4 } \\
\hline Clinical lymph node status & $2(2.5 \%)$ & $77(97.5 \%)$ & 0.001 \\
\hline NO & $36(6.6 \%)$ & $507(93.4 \%)$ & \\
\hline N1 & $52(12.2 \%)$ & $373(87.8 \%)$ & \\
\hline \multicolumn{4}{|l|}{ N2 } \\
\hline SBR grade & $2(2.6 \%)$ & $75(97.4 \%)$ & 0.007 \\
\hline 1 & $32(7.9 \%)$ & $373(92.1 \%)$ & \\
\hline 2 & $53(11.4 \%)$ & $413(88.6 \%)$ & \\
\hline 3 & $3(3.0 \%)$ & $96(97.0 \%)$ & \\
\hline \multicolumn{4}{|l|}{ unknown } \\
\hline Histologic type & $87(8.5 \%)$ & $941(91.5 \%)$ & 0.052 \\
\hline Invasive ductal carcinoma & $2(20.0 \%)$ & $8(80.0 \%)$ & \\
\hline Invasive lobular carcinoma & $0(0 \%)$ & $4(100 \%)$ & \\
\hline Mucinous carcinoma & $1(20 \%)$ & $4(80.0 \%)$ & \\
\hline \multicolumn{4}{|l|}{ Others } \\
\hline ER & $40(6.3 \%)$ & $594(93.7 \%)$ & 0.001 \\
\hline positive & $50(12.1 \%)$ & $363(87.9 \%)$ & \\
\hline
\end{tabular}

ER, estrogen receptor; HR, hormone receptor; HER-2, human epidermal growth factor receptor 2; LRR, locoregional recurrence; NAC, neoadjuvant chemotherapy; PCR, pathological complete response; PR, progesterone receptor 


\begin{tabular}{|c|c|c|c|}
\hline Parameter & LRR & No LRR & p-value \\
\hline PR & $31(5.9 \%)$ & $496(94.1 \%)$ & 0.002 \\
\hline positive & $59(11.3 \%)$ & $461(88.7 \%)$ & \\
\hline \multicolumn{4}{|l|}{ negative } \\
\hline HER2 & $44(9.4 \%)$ & $422(90.6 \%)$ & 0.38 \\
\hline positive & $46(7.9 \%)$ & $535(92.1 \%)$ & \\
\hline \multicolumn{4}{|l|}{ negative } \\
\hline Subtype & $26(6.2 \%)$ & $393(93.8 \%)$ & 0.03 \\
\hline $\mathrm{HR}+/ \mathrm{HER} 2-$ & $18(7.6 \%)$ & $219(92.4 \%)$ & \\
\hline $\mathrm{HR}+/ \mathrm{HER} 2+$ & $26(11.4 \%)$ & $203(88.6 \%)$ & \\
\hline HR-/HER2+ & $20(12.3 \%)$ & $142(87.7 \%)$ & \\
\hline \multicolumn{4}{|l|}{ HR-/HER2- } \\
\hline Neoadjuvant Regimens & $6(16.2 \%)$ & $31(83.8 \%)$ & 0.28 \\
\hline Anthracycline only & $5(6.1 \%)$ & $77(93.9 \%)$ & \\
\hline Taxane only & $54(9.0 \%)$ & $549(91.0 \%)$ & \\
\hline Anthracycline + Taxane & $25(7.7 \%)$ & $300(92.3 \%)$ & \\
\hline \multicolumn{4}{|c|}{ Chemotherapy + Target therapy } \\
\hline Operation type & $55(8.9 \%)$ & $560(91.1 \%)$ & 0.63 \\
\hline Mastectomy & $35(8.1 \%)$ & $397(91.9 \%)$ & \\
\hline \multicolumn{4}{|l|}{ BCS } \\
\hline $\mathrm{pCR}$ & $9(3.9 \%)$ & $223(96.1 \%)$ & 0.004 \\
\hline yes & $81(9.9 \%)$ & $734(90.1 \%)$ & \\
\hline \multicolumn{4}{|l|}{ no } \\
\hline $\begin{array}{l}\text { ER, estrogen receptor; HR } \\
\text { locoregional recurrence; } \\
\text { progesterone receptor }\end{array}$ & $\begin{array}{l}-2 \text {, human } \\
\text { ptherapy; } p\end{array}$ & $\begin{array}{l}\text { I growth fac } \\
\text { logical com }\end{array}$ & $\begin{array}{l}\text { tor 2; LRR } \\
\text { sponse; PR }\end{array}$ \\
\hline
\end{tabular}


Table 5

Multivariate analysis of factors to predict LRR

\begin{tabular}{|c|c|c|c|}
\hline Parameter & Odds ratio & $95 \%$ confidence interval & p-value \\
\hline Clinical T stage & 1 & $0.163-1.275$ & 0.134 \\
\hline T1 & 0.455 & $0.164-1.505$ & 0.216 \\
\hline $\mathrm{T} 2$ & 0.497 & $0.215-1.831$ & 0.394 \\
\hline T3 & 0.628 & & \\
\hline \multicolumn{4}{|l|}{ T4 } \\
\hline Clinical lymph node status & 1 & $0.579-10.675$ & 0.220 \\
\hline No & 2.487 & $0.920-17.017$ & 0.065 \\
\hline N1 & 3.956 & & \\
\hline \multicolumn{4}{|l|}{ N2 } \\
\hline SBR grade & 1 & $0.666-12.418$ & 0.157 \\
\hline 1 & 2.876 & $0.863-16.003$ & 0.078 \\
\hline 2 & 3.716 & $0.227-9.523$ & 0.687 \\
\hline 3 & 1.469 & & \\
\hline \multicolumn{4}{|l|}{ unknown } \\
\hline Subtype & 1 & $0.782-2.829$ & 0.226 \\
\hline HR+/HER2- & 1.488 & $1.117-3.829$ & 0.021 \\
\hline HR+/HER2+ & 2.068 & $1.210-4.435$ & 0.011 \\
\hline HR-/HER2+ & 2.316 & & \\
\hline \multicolumn{4}{|l|}{ HR-/HER2- } \\
\hline $\mathrm{pCR}$ & 1 & $1.308-5.999$ & 0.008 \\
\hline Yes & 2.801 & & \\
\hline \multicolumn{4}{|l|}{ No } \\
\hline
\end{tabular}

In patients who underwent BCS, a significant difference was found in the LRR between the $\mathrm{pCR}$ and nonpCR groups $(p<0.05)$. The LRR of the pCR group was $4.3 \%(n=6)$ compared with $10 \%(n=29)$ in the nonpCR group (Table 6). LRRFS was also found to be significantly different between the pCR and non-pCR groups, as shown in Fig. 3 ( $p=0.033$ ). Further analysis focusing on the subtypes of the BCS group 
demonstrated that the HR-/HER2 + non-pCR group had significantly increased LRR compared with the HR-/HER2 + pCR group (22.2\% vs 6.3\%, p < 0.05). Furthermore, the HR-/HER2-non-pCR group had significantly increased LRR compared with the HR-/HER2-pCR group ( $0 \%$ vs $20.4 \%, p<0.005)$. (Table 7 ). Moreover, there was no difference in LRR found with regards to hormone receptor positive disease between the between p-CR and non-pCR groups. In the mastectomy group $(n=615), p C R$ remained an independent factor for favorable LRR compared with non-pCR patients $(p=0.041)$, with 3 patients $(3.3 \%)$ in the pCR group developing LRR compared with 52 patients $(9.9 \%)$ in non-pCR group (Table 6). Further analysis of molecular subtypes indicated no significant difference for all subtypes between the $\mathrm{pCR}$ and non-pCR groups following mastectomy (Table 7). The LRRFS rate in the pCR group following mastectomy showed a favorable outcome; however, this was not significant when compared with the non-pCR group $(p=0.060)$ (Fig. 4). The significant difference of LRRFS rate was also shown in the $p C R$ group compared with the non-pCR group in HR-/HER2 + and HR-/HER2- patients who underwent BCS (Fig. 5-6).

Table 6

Comparison of LRR in pCR and non-pCR patients after BCS and mastectomy

\begin{tabular}{|c|c|c|c|c|c|}
\hline \multirow[t]{3}{*}{ Parameters } & & \multirow{3}{*}{$\begin{array}{l}\text { Recurrence } \\
\text { Patterns }\end{array}$} & \multicolumn{3}{|c|}{ Pathological total response } \\
\hline & & & \multirow[t]{2}{*}{$\mathrm{pCR}(\mathrm{n}=232)$} & Non-pCR & \multirow[t]{2}{*}{$\mathrm{p}$-value } \\
\hline & & & & $(n=815)$ & \\
\hline \multirow{4}{*}{$\begin{array}{l}\text { Operation } \\
\text { type }\end{array}$} & \multirow[t]{2}{*}{ Mastectomy $(n=615)$} & LRR & $3(3.3 \%)$ & $52(9.9 \%)$ & \multirow[t]{2}{*}{0.041} \\
\hline & & No LRR & $88(96.7 \%)$ & $472(90.1 \%)$ & \\
\hline & \multirow[t]{2}{*}{$\operatorname{BCS}(n=432)$} & LRR & $6(4.3 \%)$ & $29(10.0 \%)$ & \multirow[t]{2}{*}{0.041} \\
\hline & & No LRR & 135 (95.7\%) & $262(90.0 \%)$ & \\
\hline
\end{tabular}


Table 7

Comparison of LRR in pCR and non-pCR patients after BCS and mastectomy in different subtypes

\begin{tabular}{|c|c|c|c|c|c|}
\hline \multicolumn{2}{|l|}{ Parameters } & \multirow{3}{*}{$\begin{array}{l}\text { Recurrence } \\
\text { patterns }\end{array}$} & \multicolumn{3}{|c|}{ Pathological total response } \\
\hline \multirow[t]{2}{*}{ Operation type } & \multirow[t]{2}{*}{ Subtype } & & $\mathrm{pCR}$ & Non-pCR & $\mathrm{p}$-value \\
\hline & & & $(\mathrm{N}=91)$ & $(N=524)$ & \\
\hline \multirow{8}{*}{$\begin{array}{l}\text { Mastectomy } \\
(\mathrm{N}=615)\end{array}$} & \multirow{2}{*}{$\begin{array}{l}\text { HR+/HER2- } \\
(\mathrm{N}=266)\end{array}$} & \multirow{2}{*}{$\begin{array}{l}\text { LRR } \\
\text { No LRR }\end{array}$} & \multirow{2}{*}{$\begin{array}{l}1(9.1 \%) \\
10(90.9 \%)\end{array}$} & $18(7.1 \%)$ & \multirow[t]{2}{*}{0.565} \\
\hline & & & & $237(92.9 \%)$ & \\
\hline & \multirow{2}{*}{$\begin{array}{l}\text { HR+/HER2+ } \\
(\mathrm{N}=130)\end{array}$} & LRR & 0 & $11(10.8 \%)$ & \multirow[t]{2}{*}{0.120} \\
\hline & & No LRR & $28(100 \%)$ & $91(89.2 \%)$ & \\
\hline & \multirow{2}{*}{$\begin{array}{l}\text { HR-/HER2+ } \\
(\mathrm{N}=145)\end{array}$} & LRR & $1(2.5 \%)$ & $14(13.3 \%)$ & \multirow[t]{2}{*}{0.068} \\
\hline & & No LRR & $39(97.5 \%)$ & $91(86.7 \%)$ & \\
\hline & \multirow{2}{*}{$\begin{array}{l}\text { HR-/HER2- } \\
(\mathrm{N}=74)\end{array}$} & LRR & $1(8.3 \%)$ & $9(14.5 \%)$ & \multirow[t]{2}{*}{$>0.999$} \\
\hline & & No LRR & $11(91.7 \%)$ & $53(85.5 \%)$ & \\
\hline \multirow{8}{*}{$\begin{array}{l}\text { Conserving breast surgery } \\
(n=432)\end{array}$} & $\mathrm{HR}+/ \mathrm{HER} 2-$ & LRR & $1(6.3 \%)$ & $6(4.4 \%)$ & \multirow[t]{2}{*}{0.546} \\
\hline & $(N=153)$ & No LRR & $15(93.7 \%)$ & $131(95.6 \%)$ & \\
\hline & \multirow{2}{*}{$\begin{array}{l}\text { HR+/HER2+ } \\
(\mathrm{N}=107)\end{array}$} & LRR & $2(5.3 \%)$ & $5(7.2 \%)$ & \multirow[t]{2}{*}{$>0.999$} \\
\hline & & No LRR & $36(94.7 \%)$ & $64(92.8 \%)$ & \\
\hline & \multirow{2}{*}{$\begin{array}{l}\text { HR-/HER2+ } \\
(\mathrm{N}=84)\end{array}$} & LRR & $3(6.3 \%)$ & $8(22.2 \%)$ & \multirow[t]{2}{*}{0.048} \\
\hline & & No LRR & $45(93.7 \%)$ & $28(77.8 \%)$ & \\
\hline & \multirow{2}{*}{$\begin{array}{l}\text { HR-/HER2- } \\
(\mathrm{N}=88)\end{array}$} & LRR & 0 & $10(20.4 \%)$ & \multirow[t]{2}{*}{0.002} \\
\hline & & No LRR & 39 (100\%) & 39 (79.6\%) & \\
\hline
\end{tabular}

HR, hormone receptor; HER-2, human epidermal growth factor receptor 2; LRR, locoregional recurrence; pCR, pathological complete response

\section{Discussion}

In total, 232 patients $(22.2 \%)$ achieved pCR. Multivariate analysis indicated that clinical T stage, clinical N stage, and molecular subtype were independent predictors to pCR. A total of 90 patients (8.6\%) developed LRR of which 9 were in the pCR group and 81 were in the non-pCR group. Our study reported that pCR in all breast cancer subtypes, after NAC, provided better local control. The result was in line with the findings of our previous report published in 2018, in which no LRR occurred in the PCR group and 31 patients $(13.2 \%)$ in the non-pCR group with significant difference in total 263 patients all receiving neoadjuvant 
weekly epirubicin and docetaxel regimens[12]. Our study revealed that 232 patients $(22.2 \%)$ achieved pCR among the 1047 patients underwent while the BCS rate is $41 \%$ and the rest of patients received mastectomy (59\%). Overall, 240 patients experienced tumor recurrence $(22.9 \%)$.

Although the pooled analysis from CTNeoBC did not support pCR as a surrogate endpoint for an improved event-free survival or overall survival in all subtypes of breast cancer subtypes[13], pCR was an effective surrogate endpoint for selected patients in aggressive subtypes including luminal B/HER-, HER2 overexpression and triple negative breast cancer[14]. A recent comprehensive meta-analysis concluded that $\mathrm{pCR}$ followed by NAC was associated with significantly better event-free survival (EFS) and overall survival (OS), especially for patients with triple-negative and HER2 + breast cancer. The tumor response effect observed in the PCR group was similar in adjuvant chemotherapy and NAC patients[15]. Moreover, data from the combined analysis of the National Surgical Adjuvant Breast and Bowel Project (NSABP) B18 and B-27 showed that the residual tumor status was an independent predictor of LRR in all patients at the 10 year follow-up, regardless of surgery type[10]. Another large analysis of the European Organisation for Research and Treatment of Cancer (EORTC) 10994/BIG 1 - 00 study of patients with locally advanced breast cancer receiving NAC showed that $\mathrm{PCR}$ was a favorable factor with regards to the prediction of LRR after NAC[16]. Several retrospective series also demonstrated that achieving pCR after NAC can result in better local control following surgery $[1-2,17-18]$. Therefore, achieving pCR was an important factor not only for distant disease control but also for local control.

The results from the EORTC 10994/BIG 1 - 00 study of patients with locally advanced breast cancer receiving NAC showed that breast cancer subtypes, including HER2 + with or without trastuzumab and triple-negative, are predictive factors for high LRR after NAC[16]. Yang et al. reported that 233 stage II-III breast cancer patients treated with NAC, mastectomy, and post-mastectomy radiotherapy had an $8 \%$ LRR rate over 5 years with a 62-month median follow-up. The authors concluded that patients with triplenegative breast cancer had the highest LRR rate and those with HR + and HER2 + breast cancer had favorable LRR rates, regardless of NAC response[18]. In other several retrospective studies, molecular subtypes including HER2 + and triple-negative also showed poor LRR in BCS patients[1, 2]. In our study, HR-/HER2 + and HR-/HER2- subtypes were found independent significant factors for the prediction of $L R R$, regardless of treatment response.

The Early Breast Cancer Trialists' Collaborative Group (EBCTCG) recently reported that NAC was associated with more frequent local recurrence than that of adjuvant chemotherapy. The 15-year local recurrence rate was reported to be $21.4 \%$ for NACT compared with $15.9 \%$ for adjuvant chemotherapy from a meta-analysis of individual patient data from 10 randomized trials with average 9 years of followup. The study group also found that patients who underwent NAC had an increased frequency of breastconserving therapy (65\%) versus those treated with adjuvant chemotherapy (49\%). The largest difference of LRR appeared in planned mastectomy patients and surgery less commonly used patients. The authors concluded tumors downsized by NAC might have higher local recurrence after BCS than tumors of the same dimensions in women who did not undergo NAC. Furthermore, the majority of patients only received anthracycline-based chemotherapy and final enrollment of patients occurred in 2002[4]. Previous 
NSABP B-27 trial reports indicated that anthracycline-based regimens with the addition of taxane were associated with higher pCR rates and better local control[19]. Moreover, neoadjuvant chemotherapy plus trastuzumab was shown to be a predictor factor for favorable long-term survival but trastuzumab cannot be used before 2002[20]. In our studies, the majority of patients received anthracycline-based regimens combined with taxane-based chemotherapy and every patient underwent appropriate surgery. Results from the combined study of NSABP B-18 and B-27 revealed that the 10-year LRR was $12.3 \%$ for patients with mastectomy and $10.3 \%$ for patients with lumpectomy plus whole breast radiotherapy, indicating no significant difference between the mastectomy and BCS groups following NAC[10]. In the I-SPY trial there was no substantial difference in LRR between the mastectomy and BCS groups, given the fact that the mastectomy group on average had higher clinical staging[21]. A higher breast conservation rate would not increase the LRR rate, which the NSABP B-18 and the EORTC studies have confirmed[22-23]. In another pooled analysis of 5500 participants, the mastectomy rate in NAC group was found to be lower than in the adjuvant chemotherapy group, without interfering the local control[24]. In our report, 615 (58.7\%) patients chose mastectomy, while $432(41.3 \%)$ received BCS. However, the choice of surgery types did not affect the LRR rate- $8.9 \%$ in mastectomy patients and $8.1 \%$ in BCS patients in the total population.

Subgroup analysis revealed 35 cases of LRR (14.3\%) following BCS, in which $4.3 \%$ achieved pCR group. Further investigation according to the molecular subtype showed that in the BCS group, HR-/HER2 + nonpCR patients had significantly increased LRR than HR-/HER2 + pCR patients and that HR-/HER2-non-pCR patients had a significantly increased LRR than HR-/HER2-pCR patients. In the mastectomy group, an increasing trend with regards to the risk of LRR in the non-pCR group was observed, but this was not significant. Caudle et al. reported that HR-/HER2 + and HR-/HER2- patients with a poor response to NAC had worse LRRFS after BCS. Furthermore, the authors found that patients with HR+/HER2- and $\mathrm{HR}+/ \mathrm{HER} 2$ + subtypes had excellent LRRFS, regardless of tumor response to NAC[1]. Another study group from Korea revealed HR-/HER2- subtypes and HER2 + without trastuzumab subtypes predicted higher rates of LRR after NAC and BCT, while A pCR was predictive of improved LRR in HR-/HER2- subtype[2]. Moreover, the I-SPY 1 Trial reported that the 5 -year local recurrence risk was $0 \%$ for mastectomy and $9 \%$ for breast conservation in patients with an excellent response to NAC while the local recurrence rate was $12 \%$ for mastectomy and $7 \%$ for breast conservation in significant residual disease [21]. Another critical point was the resection area of operation after NAC. In patients who undergo NAC in order to achieve breast-tumor downstaging to enable BCS, the tumor site should be marked with a clip before initiating NAC, and resection of the entire volume of breast tissue originally occupied by tumor is not necessary[25]. However, the difference of resection area influencing local recurrence was still unknown in neoadjuvant setting. Local control appeared to be worse in HR-subtype non-pCR BCS patients after NAC in our study, but the effect on overall survival remains unknown. Further investigation is needed to determine overall survival outcomes.

\section{Limitation}


One of the limitations of this study is that it was a retrospective study from a single institution, which may result in selection bias. This study is also limited by the relatively short follow-up period.

Furthermore, not all patients with the same subtype diagnosis received the same chemotherapy regimens

\section{Conclusion}

Pathological response after NAC is related to the risk of developing LRR. The LRR rate was found to be higher in non-pCR patients after NAC, especially in hormone receptor-negative patients who underwent BCS. Therefore, both the pathological response and molecular subtype should be carefully considered when choosing candidates for BCS after NAC. Some strategies to adequate surgery needed to be suggested in BCS patients after NAC, including careful tumor localization, detailed pathological assessment, evaluation of the resection area, and the margin status.

\section{Abbreviations}

NAC, Neoadjuvant chemotherapy; BCS, breast conservation surgery; LRR, locoregional recurrence; pCR, pathological complete response; HR, hormone receptor; HER2, human epidermal growth factor receptor

\section{Declarations}

\section{Ethics approval and consent to participate:}

This study was approved by ethics committee of our institution and institutional review board (IRB) number was 1711150042 . The requirement for patient informed consent was waived according to the IRB by Chang Gung Medical Foundation.

\section{Consent for publication:}

Not applicable.

\section{Availability of data and materials:}

The datasets used and/or analysed during the current study are available from the corresponding author on reasonable request.

\section{Competing interests:}

The authors declare that they have no competing interests.

\section{Funding:}


This study was supported by research plan in Chang Gung Memorial Hospital, study number CMRPG1K0061, including the support of data collection and the statisticians.

\section{Authors' contributions:}

$\mathrm{HC}, \mathrm{WC}$ and RD analyzed and interpreted the patient data, and were major contributors in writing the manuscript and performed the analysis of statistics; YL and SC supervised the manuscript. Other authors collected the clinical data. All authors read and approved the final manuscript

\section{Acknowledgements:}

Not applicable.

\section{References}

1. Caudle AS. Yu TK, Tucker S. Bedrosian I, Litton JK. Gonzalez-Angulo AM, et al., Local-regional control according to surrogate markers of breast cancer subtypes and response to neoadjuvant chemotherapy in breast cancer patients undergoing breast conserving therapy. Breast Cancer Res 2012;14(3):R83.

2. Jwa E. Shin KH, Kim JY. Park YH, Jung SY. Lee ES, et al., Locoregional recurrence by tumor biology in breast cancer patients after preoperative chemotherapy and breast conservation treatment. Cancer Res Treat 2016;48(4):1363-72.

3. Veiga DF, Campos FSM. Ribeiro LM, Archangelo I Jr, Veiga Filho J. Juliano Y, et al., Mastectomy versus conservative surgical treatment: the impact on the quality of life of women with breast cancer. Revista Brasileira de Saúde Materno Infantil 2010;10:1-57.

4. Asselain B. Barlow W, Bartlett J. Early Breast Cancer Trialists' Collaborative G. Long-term outcomes for neoadjuvant versus adjuvant chemotherapy in early breast cancer: meta-analysis of individual patient data from ten randomised trials. Lancet Oncol 2018;9(1):27-39.

5. Mauri D, Pavlidis N. loannidis JPA, Neoadjuvant versus adjuvant systemic treatment in breast cancer: A meta-analysis. JNCI 2005;97(3):188 - 94.

6. van Dongen JA, Voogd AC. Fentiman IS, Legrand C. Sylvester RJ, Tong D et al., Long-term results of a randomized trial comparing breast-conserving therapy with mastectomy: European Organization for Research and Treatment of Cancer 10801 Trial. JNCI 2000;92(14):1143-50..

7. Veronesi U. Cascinelli N, Mariani L. Greco M, Saccozzi R. Luini A, et al., Twenty-year follow-up of a randomized study comparing breast-conserving surgery with radical mastectomy for early breast cancer. NEJM 2002;347(16):1227-32.

8. Sun Y. Liao MJ, He L. Zhu CF, Comparison of breast-conserving surgery with mastectomy in locally advanced breast cancer after good response to neoadjuvant chemotherapy: A PRISMA-compliant 
systematic review and meta-analysis. Medicine 2017;96(43):8367.

9. Zhou X, Li Y, Local recurrence after breast-conserving surgery and mastectomy following neoadjuvant chemotherapy for locally advanced breast cancer - a meta-analysis. Breast Care (Basel) 2016;11(5):345 - 51.

10. Mamounas EP. Anderson SJ, Bear HD. Julian TB, Geyer CE Jr, Taghian A, et al., Predictors of locoregional recurrence after neoadjuvant chemotherapy: results from combined analysis of National Surgical Adjuvant Breast and Bowel Project B-18 and B-27. J Clin Oncol 2012;30(32):39606.

11. Matsuda N. Hayashi N, Ohde S. Yagata H, Kajiura Y. Yoshida A, et al., A nomogram for predicting locoregional recurrence in primary breast cancer patients who received breast-conserving surgery after neoadjuvant chemotherapy. J Surg Oncol 2014;109(8):764-9.

12. Chou HH. Kuo WL, Yu CC. Tsai HP, Shen SC. Chu CH, et al., Impact of age on pathological complete response and locoregional recurrence in locally advanced breast cancer after neoadjuvant chemotherapy. Biomed J 2019;42:66-74.

13. Cortazar P. Zhang L, Untch M. Mehta K, Costantino JP. Wolmark N, et al., Pathological complete response and long-term clinical benefit in breast cancer: the CTNeoBC pooled analysis. Lancet 2014;384:164 - 72.

14. von Minckwitz G, Untch M. Blohmer JU, Costa SD. Eidtmann H, Fasching PA. Definition and impact of pathologic complete response on prognosis after neoadjuvant chemotherapy in various intrinsic breast cancer subtypes. J Clin Oncol 2012;30:1796 - 804.

15. Spring LM. Fell G, Afre A. Sharma C, Greenup RA. Reynolds KL, et al., Pathological complete response after neoadjuvant chemotherapy and impact on breast cancer recurrence and survival: a comprehensive meta-analysis. Clin Cancer Res 2020;26:2838-48.

16. Gillon P. Touati N, Breton-Callu C. Slaets L, Cameron D. Bonnefoi H. Factors predictive of locoregional recurrence following neoadjuvant chemotherapy in patients with large operable or locally advanced breast cancer: An analysis of the EORTC 10994/BIG 1 - 00 study. Eur J Cancer 2017;79:226 - 34.

17. Swisher SK. Vila J, Tucker SL. Bedrosian I, Shaitelman SF. Litton JK, et al., Locoregional control according to breast cancer subtype and response to neoadjuvant chemotherapy in breast cancer patients undergoing breast-conserving therapy. Ann Surg Oncol 2016;23:749 - 56.

18. Yang TJ. Morrow M, Modi S. Zhang Z, Krause K. Siu C, et al., The effect of molecular subtype and residual disease on locoregional recurrence in breast cancer patients treated with neoadjuvant chemotherapy and postmastectomy radiation. Ann Surg Oncol 2015;22:495-501.

19. Bear HD. Anderson S, Smith RE. Geyer CE Jr, Mamounas EP, Fisher B, et al., Sequential preoperative or postoperative docetaxel added to preoperative doxorubicin plus cyclophosphamide for operable breast cancer: National Surgical Adjuvant Breast and Bowel Project protocol B-27. J Clin Oncol 2006;24: 2019-27.

20. Untch M. Fasching PA, Konecny GE. Hasmüller S, Lebeau A. Kreienberg R, et al., Pathologic complete response after neoadjuvant chemotherapy plus trastuzumab predicts favorable survival in human 
epidermal growth factor receptor 2-overexpressing breast cancer: results from the TECHNO trial of the AGO and GBG study groups. J Clin Oncol 2011;29: 3351-7.

21. Cureton EL. Yau C, Alvarado MD. Krontiras H, Ollila DW. Ewing CA, et al., Local recurrence rates are low in high-risk neoadjuvant breast cancer in the I-SPY 1 Trial (CALGB 150007/150012; ACRIN 6657). Ann Surg Oncol 2014;21:2889-96.

22. Buchholz TA. Mittendorf EA, Hunt KK. Surgical considerations after neoadjuvant chemotherapy: breast conservation therapy. J Natl Cancer Inst Monogr 2015;2015:11 - 4.

23. van der Hage JA, van de Velde CJ, Julien JP. Tubiana-Hulin M, Vandervelden C. Duchateau L. Preoperative chemotherapy in primary operable breast cancer: results from the European Organization for Research and Treatment of Cancer trial 10902. J Clin Oncol 2001;19(22):4224-37.

24. Mieog JS. van der Hage JA, van de Velde CJ, Neoadjuvant chemotherapy for operable breast cancer. Br J Surg. 2007;94;1189 - 200.

25. Boughey JC. Peintinger F, Meric-Bernstam F. Perry AC, Hunt KK. Babiera GV, et al. Impact of preoperative versus postoperative chemotherapy on the extent and number of surgical procedures in patients treated in randomized clinical trials for breast cancer. Ann Surg 2006;244;464 - 70.

\section{Figures}

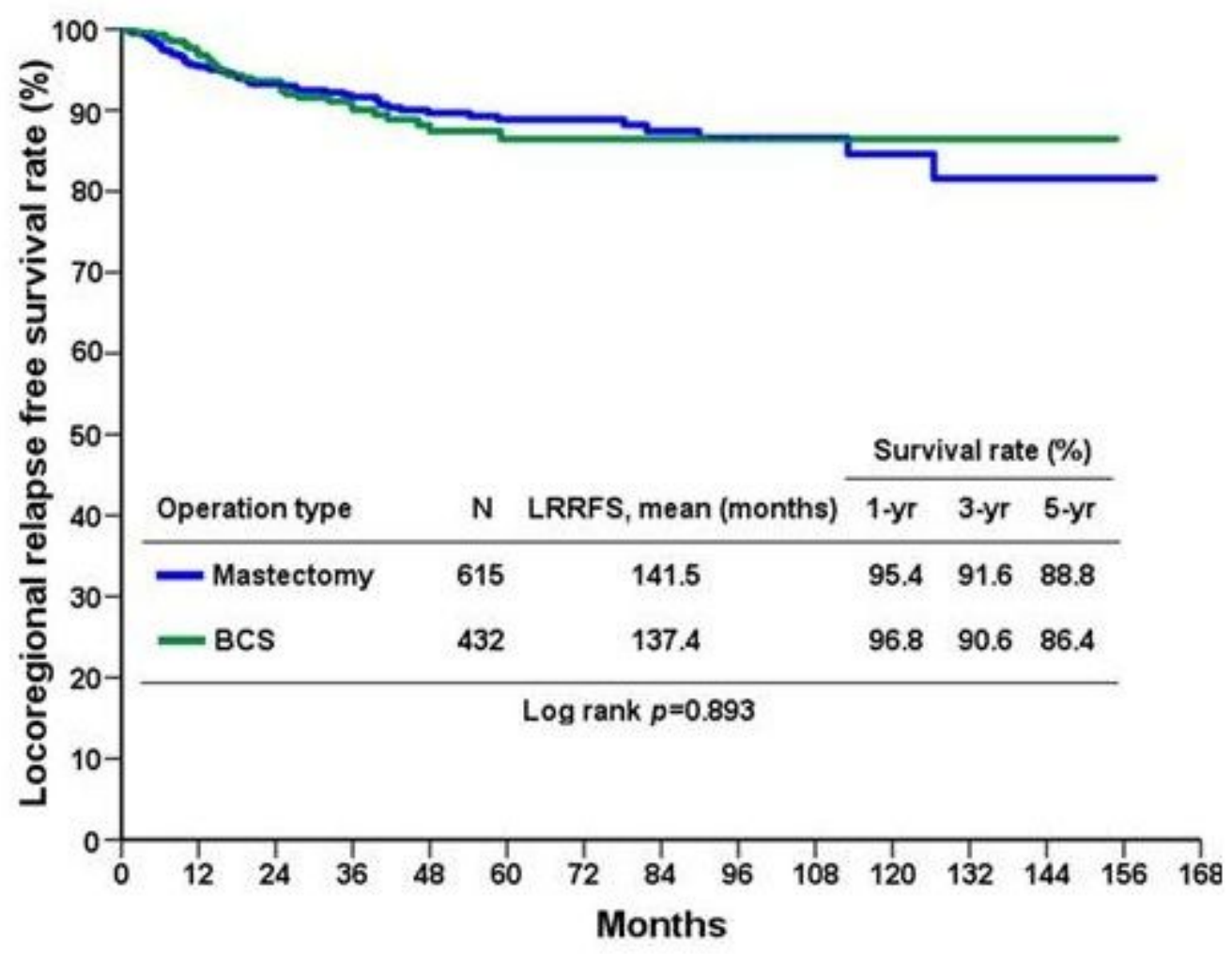

Figure 1 
LRRFS rate in the BCS group compared with the mastectomy group.

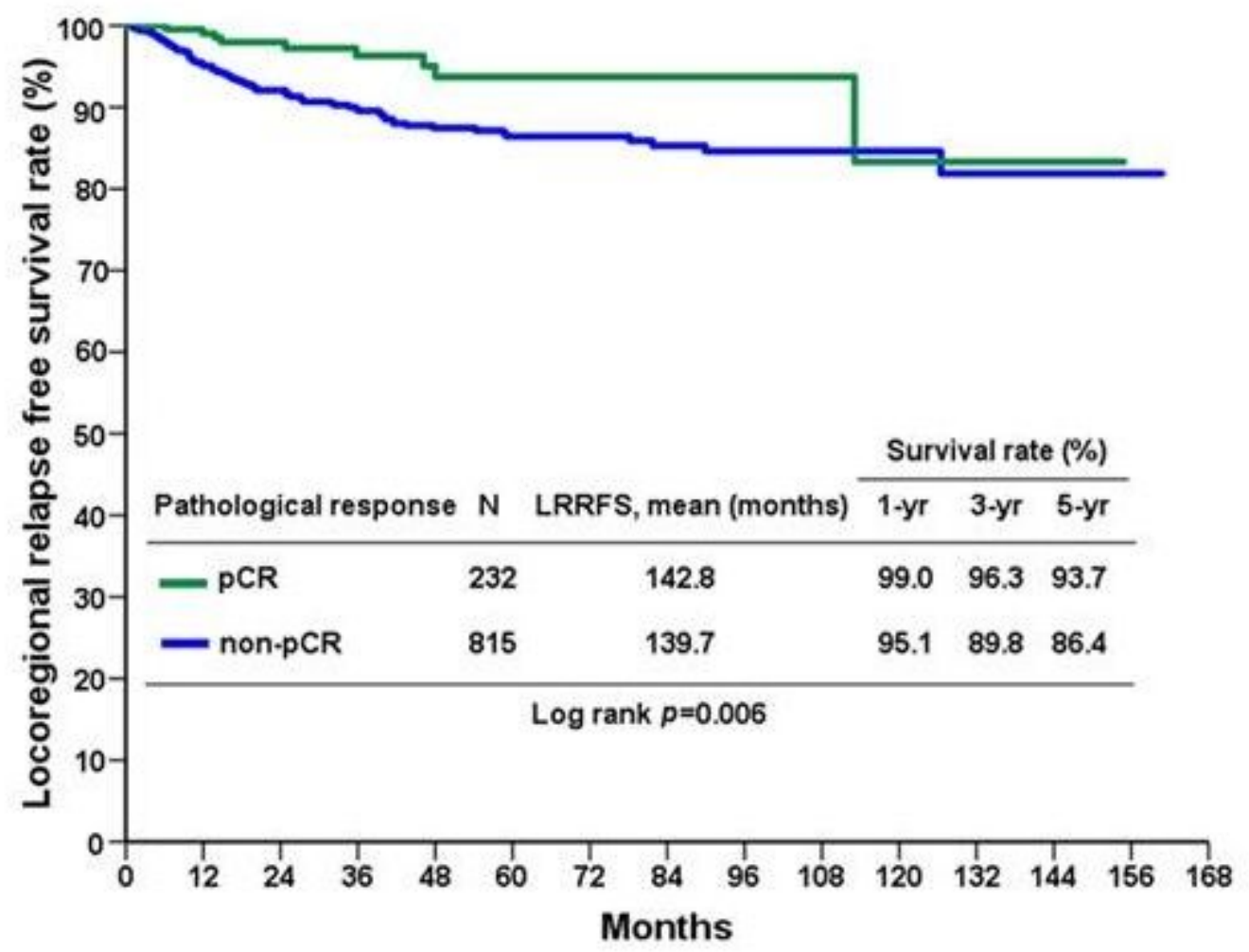

Figure 2

LRRFS rate in the pCR group compared with the non-pCR group. 


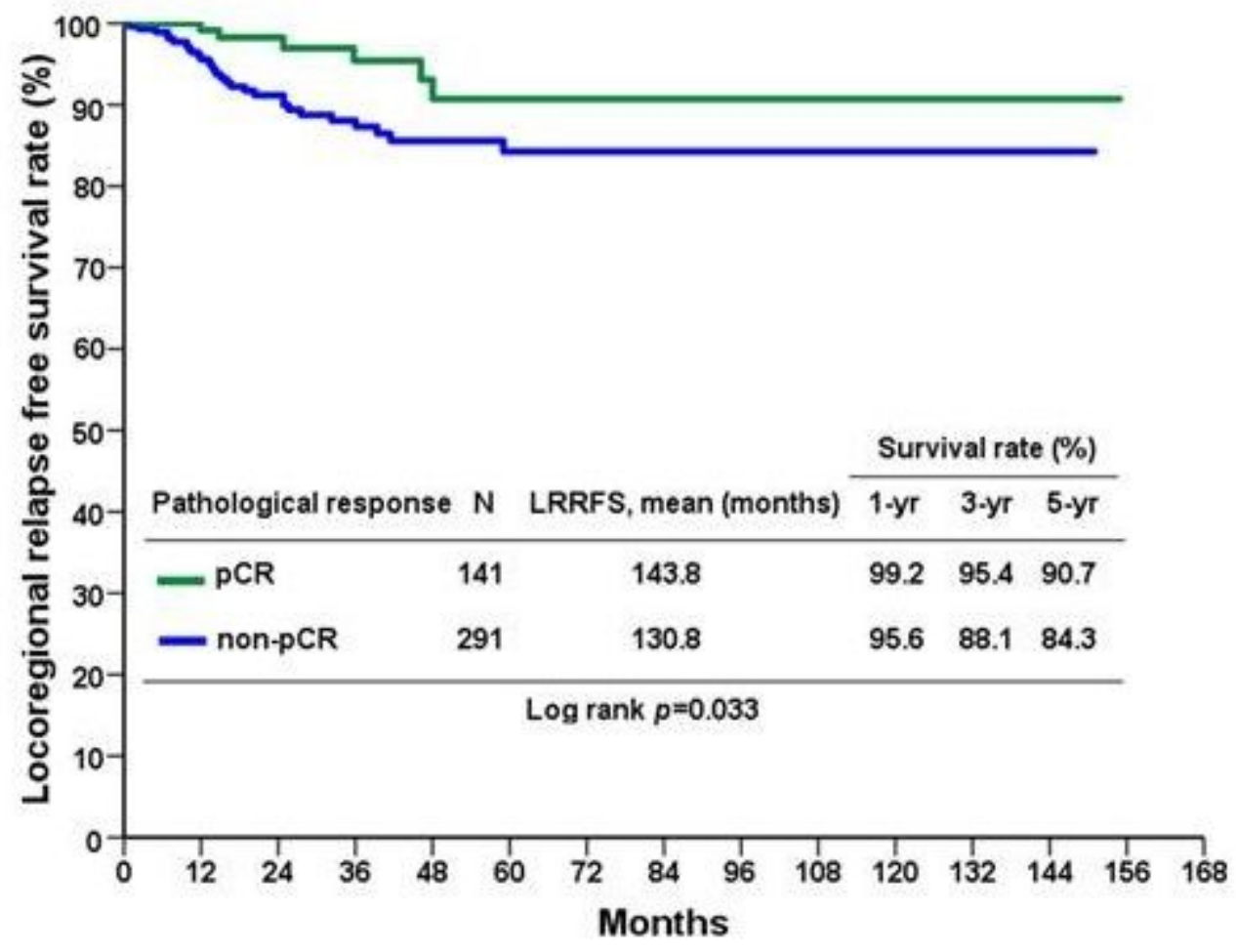

Figure 3

LRRFS rate in the pCR group compared with the non-pCR group in patients who underwent BCS. 


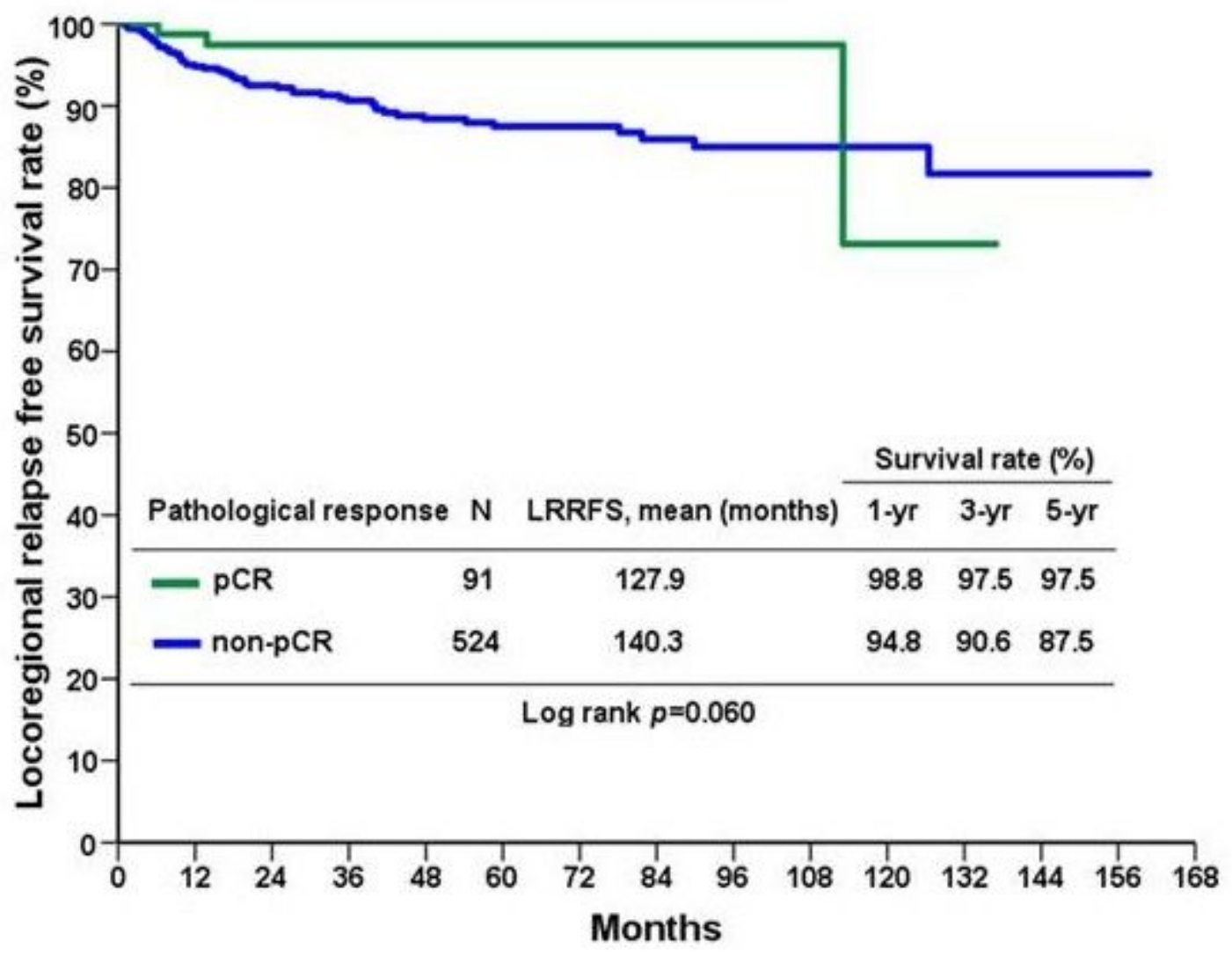

Figure 4

LRRFS rate in the pCR group compared with the non-pCR group in patients who underwent mastectomy. 


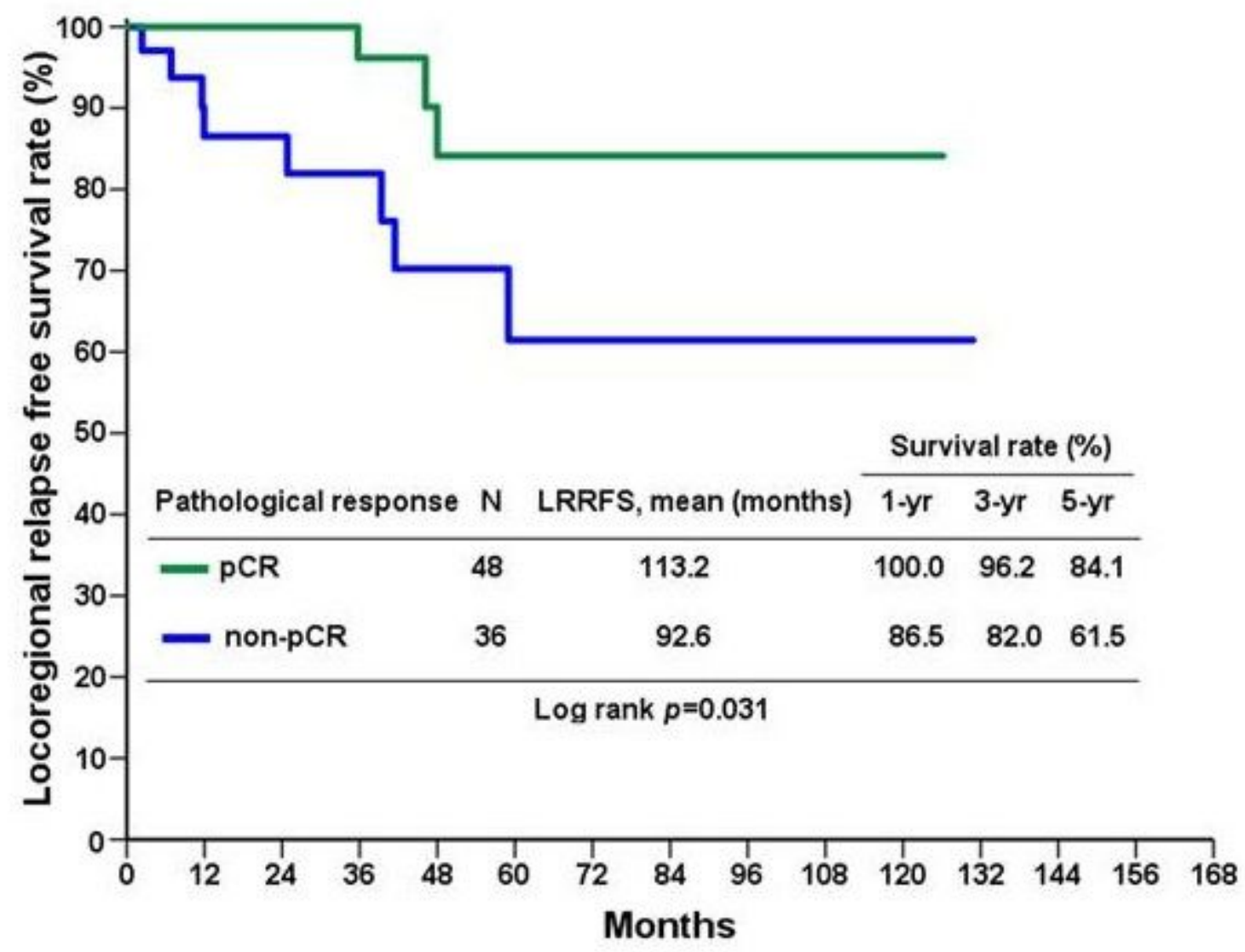

Figure 5

LRRFS rate in the pCR group compared with the non-pCR group in HR-/HER2+ patients who underwent BCS. 


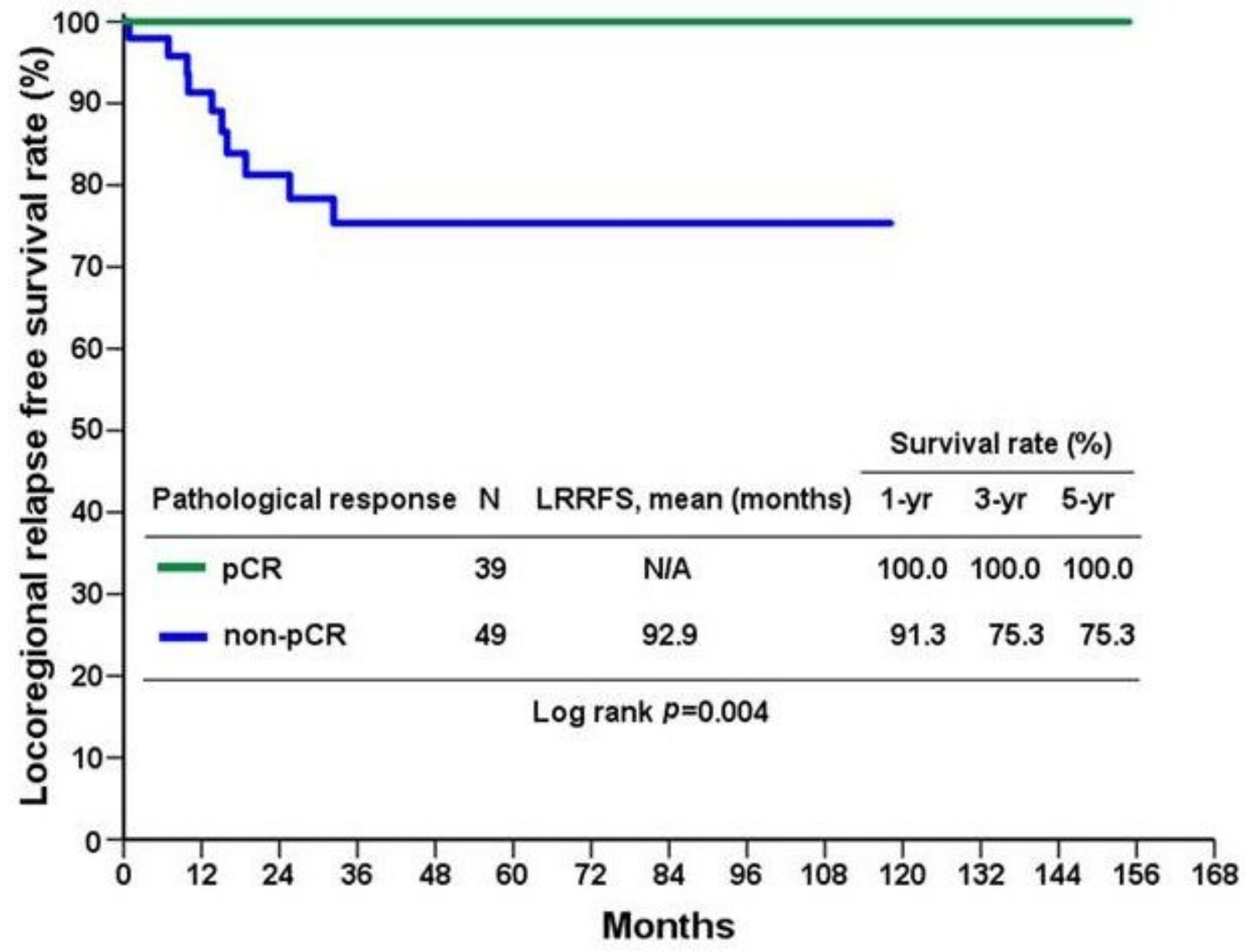

Figure 6

LRRFS rate in the pCR group compared with the non-pCR group in HR-/HER2- patients who underwent BCS. 